\title{
Tracing thyroid hormone-disrupting compounds: database compilation and structure-activity evaluation for an effect-directed analysis of sediment
}

\author{
Jana M. Weiss ${ }^{1}$ - Patrik L. Andersson ${ }^{2} \cdot$ Jin Zhang ${ }^{2}$ - Eszter Simon ${ }^{1,3}$. \\ Pim E. G. Leonards ${ }^{1} \cdot$ Timo Hamers $^{1} \cdot$ Marja H. Lamoree ${ }^{1}$
}

Received: 10 February 2015 /Revised: 17 April 2015 / Accepted: 23 April 2015 /Published online: 19 May 2015

(C) The Author(s) 2015. This article is published with open access at Springerlink.com

\begin{abstract}
A variety of anthropogenic compounds has been found to be capable of disrupting the endocrine systems of organisms, in laboratory studies as well as in wildlife. The most widely described endpoint is estrogenicity, but other hormonal disturbances, e.g., thyroid hormone disruption, are gaining more and more attention. Here, we present a review and chemical characterization, using principal component analysis, of organic compounds that have been tested for their capacity to bind competitively to the thyroid hormone transport protein transthyretin (TTR). The database contains 250 individual compounds and technical mixtures, of which 144 compounds are defined as TTR binders. Almost one third of these compounds $(n=52)$ were even more potent than the natural hormone thyroxine $\left(\mathrm{T}_{4}\right)$. The database was used as a tool to assist in the identification of thyroid hormone-disrupting compounds (THDCs) in an effect-directed analysis (EDA) study of a sediment sample. Two compounds could be confirmed to contribute to the detected TTR-binding potency in the sediment sample, i.e., triclosan and nonylphenol technical mixture. They constituted less than $1 \%$ of the TTR-binding potency of the unfractionated extract. The low rate of explained
\end{abstract}

Electronic supplementary material The online version of this article (doi:10.1007/s00216-015-8736-9) contains supplementary material, which is available to authorized users.

Jana M. Weiss

jana.weiss@vu.nl

1 Institute for Environmental Studies (IVM), Faculty of Earth and Life Sciences, VU University, De Boelelaan 1087, 1081HV Amsterdam, The Netherlands

2 Chemistry Department, Umeå University, 90187 Umeå, Sweden

3 Present address: Oekotoxzentrum, Eawag, BU F 15-19, Überlandstrasse 133, 8600 Dübendorf, Switzerland activity may be attributed to the challenges related to identification of unknown contaminants in combination with the limited knowledge about THDCs in general. This study demonstrates the need for databases containing compoundspecific toxicological properties. In the framework of EDA, such a database could be used to assist in the identification and confirmation of causative compounds focusing on thyroid hormone disruption.

Keywords Thyroid hormone-disrupting compound(THDC) · Transthyretin (TTR) $\cdot$ Database $\cdot$ Structure-activity relationship (SAR) · Effect-directed analysis (EDA) . Sediment

\section{Introduction}

Both anthropogenic and naturally occurring compounds that are widely spread in the environment have the potential to disrupt the endocrine system of organisms. Reported impacts of endocrine-disrupting compounds (EDCs) in wildlife are, e.g., decreased fertility, altered masculinity and femininity, compromised immune system, gross birth defects, and thyroid dysfunctions [1]. Thyroid hormones (TH) play an important role in the (embryonic) development and in the maintenance of a normal physiological state. Compounds in the environment that have THdisrupting properties could have devastating effects on individuals as well as on whole populations. Therefore, it is important to identify thyroid hormone-disrupting compounds (THDCs) and to construct screening methodologies to be included in monitoring programs [2].

TH disruption can be caused through many different pathways, such as interference with TH metabolism, TH excretion, or $\mathrm{TH}$ transport [3-5]. In 2006, a review paper on $\mathrm{TH}$ 
disruption assays was published by the Organisation for Economic Co-operation and Development (OECD) [3], and in 2010, it was proposed to establish a thyroid scoping effort group (TSEG) to determine the state of in vitro thyroid assays since 2006. The purpose of the TSEG was to bring relevant in vitro thyroid assays to the attention of OECD member countries and provide recommendations for their development and use [2]. Out of the 18 assays reviewed, the top three of the selected assays focused on TH disruption via transport protein binding [3]. Available in vitro assays identifying THDCs on a mechanism-base have been thoroughly discussed in a recently published review [6]. This study also recommended using disrupted circulation and transport of $\mathrm{TH}$ via the binding to serum transport proteins for the evaluation of the TH-disrupting potency of compounds.

Thyroid hormones are lipophilic compounds that are poorly soluble in aqueous media, including blood plasma. Therefore, TH transport proteins that are present in the plasma are important as they increase the blood carrying capacity for TH. $\mathrm{TH}$ transport proteins are themselves regulated by $\mathrm{TH}$; thus, compounds that change circulating levels of $\mathrm{TH}$ are also likely to alter the transport protein concentration which will further change the dynamics of this endocrine system $[3,6]$. Vertebrates typically have three major $\mathrm{TH}$ transport proteins, i.e., transthyretin (TTR), thyroxine-binding globulin (TBG), and albumin. In general, these TH transport proteins have higher affinity for the precursor TH hormone thyroxine $\left(\mathrm{T}_{4}\right)$, which is therefore considered as the transport form of $\mathrm{TH}$, than for 3,3', 5-triiodothyronine $\left(\mathrm{T}_{3}\right)$, which is the active form of $\mathrm{TH}$ after deiodination of $\mathrm{T}_{4}$. Although mammalian TTR indeed has higher affinity for $\mathrm{T}_{4}$, TTR from teleost fish, amphibians, reptiles, and birds has higher affinity for $\mathrm{T}_{3}$ than for $\mathrm{T}_{4}$ [7]. Also, the relative importance of the three TH transporter proteins differs per species. Whereas TBG carries about $75 \%$ of the TH in humans, TTR is the most important TH-transporting protein in rodents, tadpoles, and fish. Albumin is a nonselective transport protein for hormones. For lower vertebrates, albumin has been suggested to be important for $\mathrm{T}_{4}$ transport, and TTR for $\mathrm{T}_{3}$ transport [8]. Binding of xenobiotics to $\mathrm{TH}$-carrying proteins is important not only because it may disrupt TH transport but also because the proteins may carry the xenobiotics to TH target tissues. This is especially the case for TTR, which can cross the placenta and the blood-brain barrier [9].

Examples of compounds that are known to interfere with $\mathrm{TH}$ transport proteins are hydroxylated polychlorinated biphenyls (OH-PCBs) and hydroxylated polybrominated diphenylethers (OH-PBDEs) [4, 10, 11]. More recently, perfluorinated compounds were reported to bind to TTR [12]. By competing with the natural ligand, TTR binders prevent proper transport of TH and cause an increase in unbound TH which is then available for further metabolization and excretion. Several different in vitro assays have described the determination of the binding potency of environmental pollutants to TTR, either based on radiolabeled ligand competitive binding (RLBA) or on the use of non-radiolabeled ligand competitive binding (BA, SPR, ANSA, and FLU-TTR). The assays are described further in detail in the Electronic Supplementary Material.

To identify TH-disrupting compounds in the environment, the TTR-binding assay can be used in effect-directed analysis (EDA). EDA involves a bioassay/effect-directed fractionation of an extract followed by chemical identification and confirmation steps. EDA was successfully applied to evaluate endocrine potencies in several water systems, e.g., in wastewater treatment plants [13-15], rivers [13,15-17], harbor areas [18, 19], marine sediments [20], and biota [21]. An earlier published review describes a range of important results from effect-directed studies, e.g., identified compounds and pinpointed hotspots [22]. Until now the main focus in EDA research has been on estrogenic and (anti-)androgenic activity and only four studies have addressed thyroid hormone disruption, two in sediment extracts from water systems, i.e., in The Netherlands [19] and in Germany [23], one in indoor dust from Japan [24], and one in polar bear plasma [25]. In polar bear plasma, the identified THDCs could successfully explain almost $80 \%$ of the observed TTR-binding potency of the extract.

A major criticism of the EDA approach is the limited identification success rate, and a key to increase its utility is to improve the availability and quality of databases containing known bioactive compounds and their chemical properties. To aid the identification of effect-causing compounds, we compiled a database that reports all compounds that have been tested in the TTR-binding assay, including both active and non-active compounds. To further explore the chemical characteristics of TTR-binding compounds, the compiled dataset was analyzed using principal component analysis (PCA) and a set of calculated chemical descriptors.

A second aim of the paper was to use the database to assist in the identification of THDCs in a sediment extract using the EDA approach [26].

\section{Methods}

\section{Database of compounds tested for their TTR-binding potency}

A database was compiled with compounds that were tested in in vitro bioassays measuring the TTR-binding potency (Table S1). The in vitro bioassays are described in the Electronic Supplementary Material. The literature was collected via ScienceDirect and PubMed, and the search was limited to compounds tested for having a direct interaction with TTR. The relative potencies, expressed in $T_{4}$ or $T_{3}$ relative effect potency (REP) values, were either extracted from the 
publications or calculated by dividing the $\mathrm{IC}_{50}$ of the natural ligand $\mathrm{T}_{4}$ by the $\mathrm{IC}_{50}$ of the competitor, with $\mathrm{IC}_{50}$ as the concentration that is capable of replacing $50 \%$ of a labeled TTR ligand from TTR. By definition, the REP of $T_{4}$ and $T_{3}$ is 1. Thus, compounds with higher affinity for TTR than the natural ligand have a REP $>1$, and compounds with lower affinity have a REP $<1$.

\section{Chemical descriptors and principal component analysis}

Of the 250 compounds in the database, 220 have been tested for their binding affinity to human TTR. These compounds were classified as binders ( $\mathrm{IC}_{50}>25 \mu \mathrm{M} ; 100$ compounds) and non-binders $\left(\mathrm{IC}_{50}>25 \mu \mathrm{M} ; 120\right.$ compounds) and characterized using 57 chemical descriptors calculated using the Molecular Operating Environment (MOE; chemcomp.com) software from their structures (represented by SMILES codes). The structures of the compounds were taken from various sources (e.g., SciFinder, PubChem) and processed by ChemAxon JChem Standardizer under the settings of "Add explicit hydrogens," "Aromatize," "Clean 2D," and "Remove fragment" (ChemAxon JChem Version 6.1.2, 2013. ChemAxon; chemaxon.com). Descriptors were selected for their chemical relevance, interpretability, and (hence) utility for describing their major chemical variation. A complete list with brief explanations can be found in the Electronic Supplementary Material (Table S2). The descriptor set includes the logarithm of the octanol-water partition coefficient $\left(\log K_{\text {ow }}\right)$, molecular polarizability, and Van der Waals volume in combination with selected flexibility, shape, and connectivity indices. In addition, molecular surface characteristics were reflected by "partial equalization or orbital electronegativity" (PEOE) descriptors. Counts of selected atom types, single and aromatic bonds, and some count ratios completed the descriptor set.

Prior to the analysis, the descriptors were logarithmically transformed (if not already log transformed) if the calculated skewness exceeded 2 to improve the normality of the descriptors' distributions and to minimize the impact of extreme values.

PCA was used to obtain an overview of the physicochemical characteristics of the compounds of the database. PCA is a latent vector-based method that compresses data into a few orthogonal vectors, principal components (PCs), summarizing the variation and correlation patterns in the data. Each PC consists of two vectors where not only similarities among objects (i.e., compounds) but also correlation patterns among the descriptors are visualized. The score vector shows similarities among the compounds, and the loading vector shows the correlation pattern among the descriptors. Here, we used the software SIMCA-P+ v13 for the multivariate analysis (umetrics.com) and an eigenvalue of 2 to define the number of significant PCs.

\section{Chemical screening of TTR binders in sediment}

The created TTR-binding database was used to identify THDC in a sediment sample from a previous EDA study. The sediment sample was collected from a polluted site in the river Schijn, a tributary to the river Scheldt, close to Antwerp (Belgium). The sample, EDA methodology, and chemical analysis have been described in detail elsewhere $[16,26]$.

In short, the sample preparation included accelerated solvent extraction, gel permeation chromatography, and fractionation by reversed-phase (RP) followed by normal-phase (NP) liquid chromatography techniques. Each RP and NP fraction was tested for its TTR-binding potency in the RLBA, described in detail elsewhere $[4,12]$. The assay has been validated in-house, showing a coefficient of variation of less than $8 \%$ over time. The limit of detection is set to $20 \%$ of the binding capacity (in the range of $16 \mathrm{nM} \mathrm{T}_{4}$ ). To improve the detection limit, the ${ }^{125} \mathrm{I}_{-} \mathrm{T}_{4}$ is always purified from free labeled iodine before incubation [12].

The identification of active compounds is facilitated by a decrease in complexity of the sample extract, which is obtained by repeated fractionation of samples with TTR-binding activity. Nevertheless, still many compounds showed to be present in the active fractions. All NP fractions were screened with GC-MS (GC Agilent 6890 with a MS, Agilent 5973), equipped with an SGE-BPX5 column ( $25 \mathrm{~m}, 0.22 \mathrm{~mm}$ i.d., $0.3 \mu \mathrm{m}$ film thickness), using splitless injection and full-scan $(m / z 50-650)$ data acquisition in electron impact (EI) mode [26], and with LC-MS using a linear quadrupole ion trapOrbitrap (LTQ-Orbitrap) mass spectrometer (Thermo Electron) with an Xbridge C18-LC column (Waters, 100× $2.1 \mathrm{~mm}, 3.5 \mu \mathrm{m}$ ) equipped with an electron spray ionization source (ESI) operating in positive mode (negative mode was evaluated but is not reported due to lack of interesting results) [16]. Survey full-scan MS spectra (from $\mathrm{m} / \mathrm{z} 50$ to 600) were acquired using the Orbitrap with a resolution of 30,000.

GC-MS spectra were deconvoluted using the Automated Mass Spectral Deconvolution and Identification System (AMDIS version 2.64) and compared with reference spectra in the National Institute of Standards and Technology (NIST version 02 ) main mass spectral database (match factor $\geq 70 \%$ ) for tentative identification [26]. The identification using LC coupled to high-resolution LTQ-Orbitrap instrumentation was described in detail elsewhere [16].

\section{Results and discussion}

\section{Chemical characterization of TTR binders}

In total, 427 entries representing 250 compounds and technical mixtures were included in the database. All compounds have been tested for their binding potency to TTR, derived 
from seven different species, human (recombinant and purified, 305 entries), birds (chicken [Gallus gallus] and gull [Larus hyperboreus and Larus argentatus], 26 entries), fishes (salmon [Onchorhynchus masou] and sea bream, 51 entries), and amphibians (Rana catesbeiana and Xenopus laevis, 45 entries).

Among the 250 compounds of the database, 222 compounds were derived from the literature and 33 compounds were tested in the present study, of which 5 have been analyzed earlier for their potency to bind to TTR. The distribution of the most common characteristics of the 250 entities is illustrated in a Venn diagram (Fig. 1); 198 compounds are halogenated (79\%); 195 contain $\geq 1$ aromatic ring system (78\%); 106 compounds are hydroxylated (42\%); 88 compounds are aromatic, halogenated, and hydoxylated (35\%); and 15 (6\%) of the compounds lack the three main characteristics, i.e., aromatic, hydroxylated, and halogenated. Based on knowledge of the structure of known environmental THDCs, halogenated aryl compounds, with a hydroxy group at a position similar to the natural ligand thyroxine $\left(\mathrm{T}_{4}\right)$, are traditionally suspected to have TTR-binding potencies. Compounds with carboxylic acids were not considered as hydroxylated because these acids are most likely not protonated under natural conditions. One hundred forty-four of the tested compounds $(58 \%)$ were able to bind to TTR, and $52(21 \%)$ were more potent than the natural hormone $\mathrm{T}_{4}(\mathrm{REP}>1)$. Among the 52 most potent compounds, 48 were aromatic, hydroxylated, and halogenated.

Some differences in the TTR-binding potency between species have been reported $[27,28]$ (see examples in Table S1). Although the primary structure of TTR has been conserved for 400 million years, some changes in amino acid residues of the N-terminal region have been identified [29].

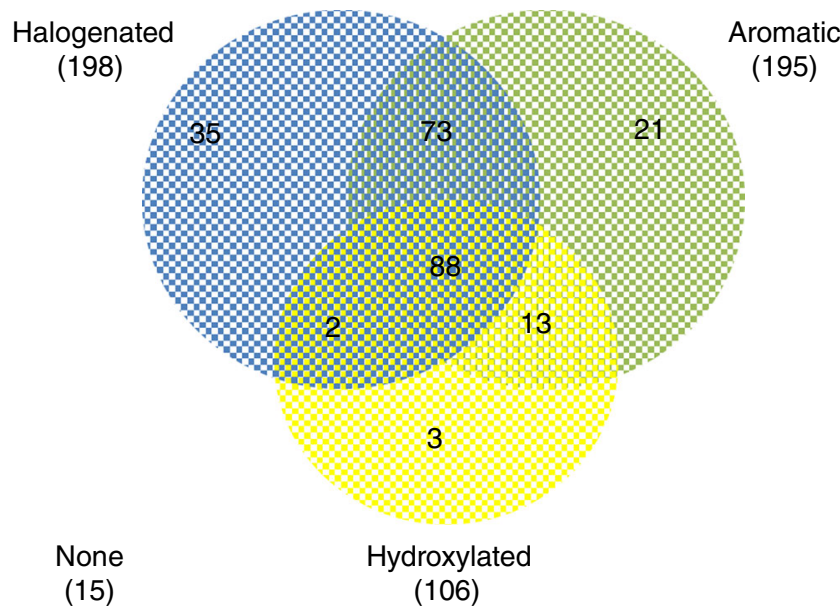

Fig. 1 A Venn diagram illustrating the distribution of the three main chemical structure characteristics, i.e., halogenation, aromaticity, and the presence of a hydroxyl group, of the 250 compounds tested in the TTR-binding assays listed in Table S1 (Electronic Supplementary Material). In brackets are the total numbers of compounds belonging to each characteristic or to none of the three characteristics
These structural modifications affect the affinity of the TTRbinding site not only to $\mathrm{T}_{4}$ but also to exogenous compounds, and explain the differences in the TTR-binding potency of compounds reported between species.

A PCA was applied to view the chemical variation of the 220 compounds tested for their binding affinity to human TTR. Four significant principal components (PCs) could explain $85 \%$ of the chemical variation, of which the first two PCs explained $64 \%$ (Fig. 2-score plot). In brief, the two first PCs display a separation into three groups covering aromatic and halogenated compounds (group 1, consisting of binders and non-binders), fatty acids (group 2, non-binders), and perfluorinated compounds (group 3, binders and non-binders). Each group is spread in the second dimension (PC2) according to polarity and molecular size (Figure S1-loading plot). The majority of active compounds is clustered (the left cluster), and these have negative PC1 values and moderate PC2 values. In this cluster, the small phenols are inactive as well as the large brominated aromatics (Fig. 2). The PCA clearly illustrates the disparate chemical properties of the perfluorinated compounds (the right cluster) in relation to the majority of active compounds which indicates dissimilar structure-activity relationships. Noteworthy are also the unique chemical profiles of the endogenous hormone $\left(\mathrm{T}_{4}\right)$ and the TTR binders tetrabromobisphenol A bis(2,3-dibromopropyl ether) TBBPA-DBPE and 4-nonylphenol (technical mixture). The reported activity of TBBPA-DBPE could, however, be due to contamination with its potent precursor TBBPA [30].

\section{Screening of THDCs in a sediment sample}

\section{TTR-binding activity in the sediment extract}

An EDA study of sediment was conducted to determine the TTR-binding potency and to identify the active compounds. Before the sediment extract was fractionated, the extract showed a total TTR-binding potency of $1000 \mathrm{pmol} \mathrm{T}_{4}$ equivalents/g dry weight (d.w.) of sediment. Using reversedphase (RP) LC, the extract was fractionated into five fractions with an increasing octanol-water partitioning coefficient $\left(K_{\mathrm{ow}}\right)$ (RP1-RP5), of which RP3 showed the highest TTR-binding affinity (i.e., 235 pmol $\mathrm{T}_{4}$ equivalents/g d.w.; first column in Fig. 3). Active RP fractions were further fractionated by normal-phase (NP) LC into eight fractions with increasing polarity. For the active RP3 fraction, this resulted in three active NP fractions, i.e., NP 5-7. Elevated TTR-binding potency was also found in RP2NP7. The summarized TTRbinding potency of the individual fractions was ca $50 \%$ of the total TTR-binding potency measured in the unfractionated extract. This lower value may be explained by the separation of active compound groups over multiple fractions, resulting in lower potencies of the individual fractions close to the limit 
Fig. 2 PCA score plot showing principal component $1(\mathrm{t} 1)$ versus 2 (t2). Compounds marked in red are reported with $\mathrm{IC}_{50}$ values $\leq 25 \mu \mathrm{M}$ and in green $>25 \mu \mathrm{M}$ in the human TTR assay. Compounds are abbreviated according to Table $\mathrm{S} 1$

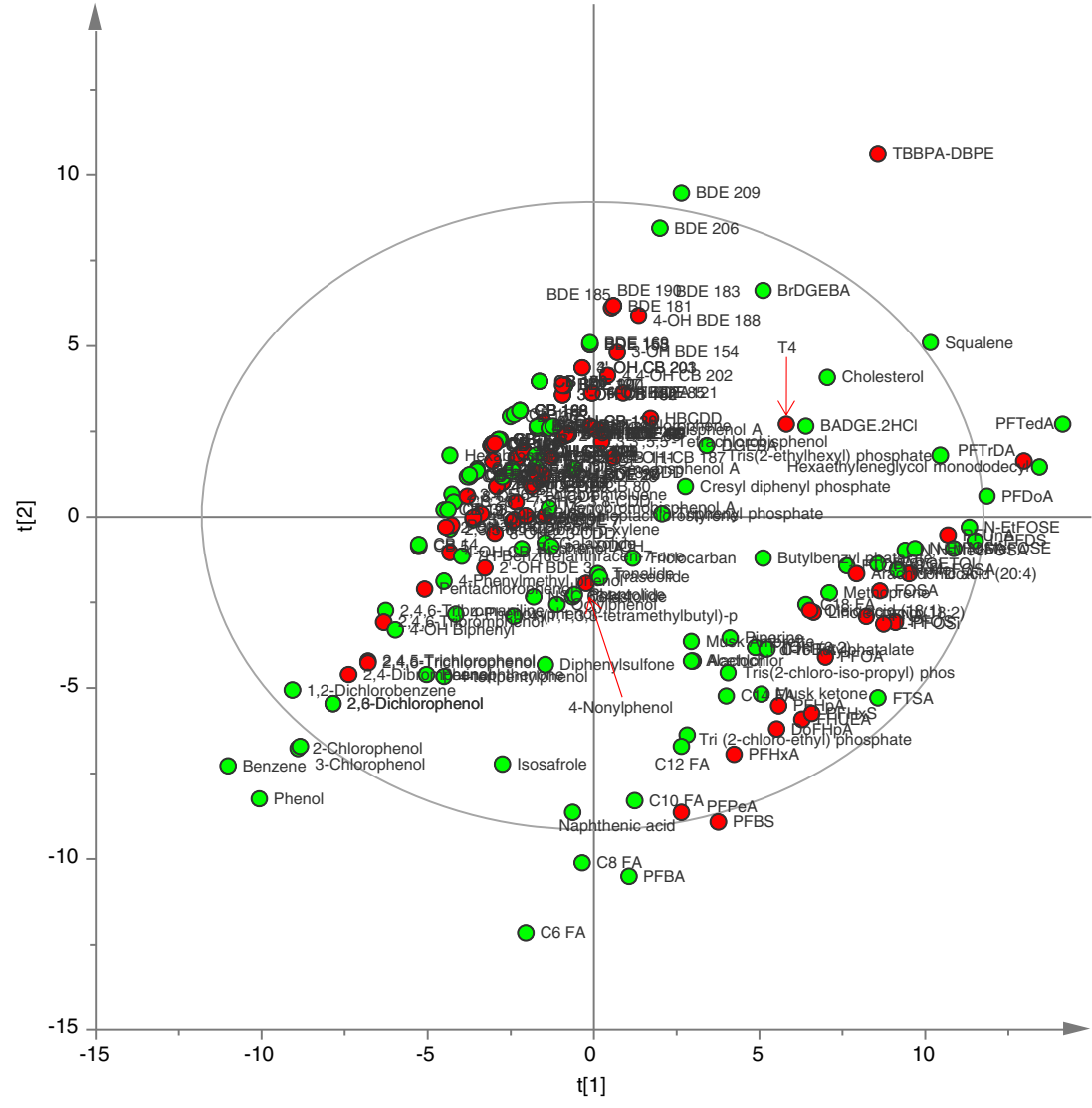

of quantification of the assay. The recovery of the potency of TTR binding was tested by pooling the NP fractions into the original composition and performing the assay again. It was confirmed that the decrease in potency after fractionation was not due to loss of compounds in the fractionation step as the initial potency was recovered in the pooled NP fractions (data not shown).

\section{Identification of THDCs in active fractions}

To identify which compounds were present in the active fractions, LTQ-Orbitrap and GC-MS analyses were carried out, from which 43 tentatively identified compounds were derived

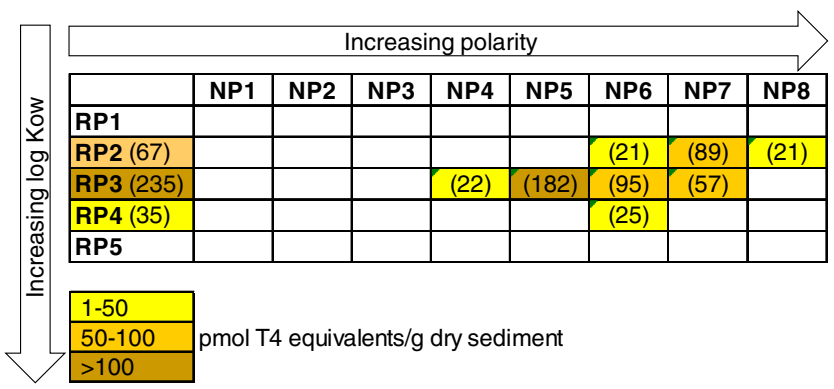

Fig. 3 The TTR-binding activity measured in the reversed-phase (RP) fractions and the normal-phase (NP) fractions. The activity (pmol $\mathrm{T}_{4}$ equivalents/g dry sediment) is given in brackets for each fraction
(Table 1). The LTQ-Orbitrap results were evaluated using the identification strategy described earlier [16]. From a total number of 6503 base peak framed masses (SIEVE), 337 accurate masses were selected for investigation. Criteria were that the peak shape was normally distributed and the mass should have an intensity 100 times higher than the adjacent control (non-active) fraction. It was not possible to perform a mass fragmentation confirmation to establish the match factor of the tentatively identified compounds in the LTQ-Orbitrap due to low intensities. Instead, the accurate mass and the isotope pattern were used for tentative identification. With this strategy, eight compounds could be tentatively identified which were present in the NIST library and then analytically confirmed by retention time using standard compounds (marked LC in Table 1).

GC-MS screening using AMDIS and the NIST library resulted in the tentative identification of 39 compounds having a $>70 \%$ match factor (marked GC in Table 1). As this was a complex matrix, mass shifts and interfering effects such as ion suppression may have occurred, possibly altering the chromatographic profile. Therefore, also compounds suggested from the NIST library search with a match factor below $70 \%$ could be of interest. Eight NIST library search indicated compounds, with a match factor between 50 and $70 \%$, are listed in Table 1, which were available in-house and tested for their TTR-binding potency. 
Table 1 Tentatively identified compounds (analyzed with GC-MS with a match factor $\geq 70 \%$ in the NIST library, or with LC-LTQ-Orbitrap on accurate mass) present in TTR-binding active fractions $(\mathrm{RP}=$ reversed-phase and $\mathrm{NP}=$ normal-phase fractionation) in a sediment sample. The span of the log
$K_{\text {ow }}$ of the RP fractions RP2, RP3, and RP4 is in the order of 2-4, 4-6, and 6-9 respectively. In addition, eight compounds indicated in the NIST library with a match factor of $50-70 \%$ and the additionally tested compounds in the TTR-binding assay are listed at the end of the table

\begin{tabular}{|c|c|c|c|c|}
\hline Fraction & CAS & Name & $\mathrm{LC} / \mathrm{GC}$ & TTR-binding activity \\
\hline \multirow[t]{6}{*}{ RP2NP6 } & $123-52-85$ & (1H-Benzoimidazol-2-yl)-diphenyl-methanol & GC & No info \\
\hline & $4237-44-9$ & 2-(1-Phenylethyl)-phenol & GC & No info \\
\hline & $23966-56-5$ & 2-Amino-3,3-diphenyl-phthalimidine & GC & No info \\
\hline & NIST-307688 & 4-Methoxyphenyl ester 2-fluorobenzoic acid & GC & No info \\
\hline & $85-68-7$ & Butylbenzyl phthalate & GC & Active [28], not active (TS) \\
\hline & $127-63-9$ & Diphenyl sulfone & GC & Not active (TS) \\
\hline \multirow[t]{8}{*}{ RP2NP7 } & 137909-40-1 & Bis(1-chloro-2-propyl)(3-chloro-1-propyl) phosphate & GC & No info \\
\hline & $115-86-6$ & Triphenyl phosphate & GC & Not active (TS) \\
\hline & $13674-84-5$ & Tris(2-chloro-iso-propyl) phosphate & GC & Not active (TS) \\
\hline & $1067-98-7$ & Tris(3-chloropropyl) phosphate & GC & No info \\
\hline & $120-58-1$ & Isosafrole & $\mathrm{LC}$ & Not active (TS) \\
\hline & $548-39-0$ & Perinaphthenone & $\mathrm{LC} / \mathrm{GC}$ & Not active (TS) \\
\hline & $959-28-4$ & (2E)-1,4-Diphenyl-2-butene-1,4-dione & $\mathrm{LC}$ & Not active (TS) \\
\hline & $94-62-2$ & Piperine & $\mathrm{LC}$ & Not active (TS) \\
\hline \multirow[t]{3}{*}{ RP3NP4 } & $225-51-4$ & Benz $[\mathrm{c}]$ acridine & GC & No info \\
\hline & $1018-97-9$ & Bis(2-methylphenyl)-methanone & GC & No info \\
\hline & $1222-05-5$ & Galaxolide & $\mathrm{LC} / \mathrm{GC}$ & Not active (TS) \\
\hline \multirow[t]{10}{*}{ RP3NP5 } & $1159-86-0$ & 1,2-Dibenzoyl benzene & GC & No info \\
\hline & $3770-82-9$ & 1,3-Dibenzoyl benzene & GC & No info \\
\hline & $3016-97-5$ & 1,4-Dibenzoylbenzene & GC & No info \\
\hline & $75694-46-1$ & 2,3-Diphenylbenzo-1,4-dioxin & GC & No info \\
\hline & $2219-84-3$ & 2-Methyl-4-(1,1,3,3-tetramethylbutyl)-phenol & GC & Not active (TS) \\
\hline & $140-66-9$ & 4-(1,1,3,3-Tetramethylbutyl)-phenol & GC & Not active (TS) \\
\hline & $80-46-6$ & 4-tert-Pentyl phenol & GC & Not active (TS) \\
\hline & $5635-50-7$ & Hexestrol & GC & No info \\
\hline & $21145-77-7$ & Tonalide & $\mathrm{LC} / \mathrm{GC}$ & Not active (TS) \\
\hline & $68140-48-7$ & Traseolide & $\mathrm{LC} / \mathrm{GC}$ & Not active (TS) \\
\hline \multirow[t]{3}{*}{ RP3NP5-6 } & $82-05-3$ & $7 H$-Benz $[d e]$ anthracen-7-one ${ }^{\mathrm{a}}$ & $\mathrm{LC}$ & Not active (TS) \\
\hline & NIST-317319 & $n$-[4-(Phenylamino)phenyl]-benzamide & GC & No info \\
\hline & $104-40-5$ & $p$-Nonylphenol technical mixture, 8 identified peaks & GC & Active (TS and [40, 25]) \\
\hline \multirow[t]{8}{*}{ RP3NP6 } & $20760-63-8$ & 1-(p-Fluorophenyl)-anthraquinone & GC & No info \\
\hline & NIST-307638 & 3,4-Dichlorophenyl ester $p$-anisic acid & $\mathrm{GC}$ & No info \\
\hline & $64436-60-8$ & 4'-Propoxy-2-methylpropiophenone & GC & No info \\
\hline & NIST-315373 & Di(3-methylphenyl) phthalate & GC & No info \\
\hline & $84-74-2$ & Di- $n$-butyl phthalate & GC & Active [28], not active (TS) \\
\hline & NIST-315190 & Ethyl hex-2-yn-4-yl phthalate & GC & No info \\
\hline & $13556-73-5$ & $n$-Benzyl- $n^{\prime}$-phenyl-p-phenylenediamine & GC & No info \\
\hline & $3380-34-5$ & Triclosan & GC & Active (TS) \\
\hline RP3NP7 & $5875-45-6$ & 2,5-Bis(1,1-dimethylethyl)-phenol & GC & No info \\
\hline \multirow[t]{4}{*}{ RP4NP6 } & $80-97-7$ & Cholestanol & GC & No info \\
\hline & $360-68-9$ & Coprostanol & GC & No info \\
\hline & $559-74-0$ & Friedelan-3-one & GC & No info \\
\hline & $1617-70-5$ & Lupenone & GC & No info \\
\hline \multicolumn{5}{|c|}{ Compounds indicated in the NIST library (match factor 50-70\%) } \\
\hline Multiple & $101-53-1$ & 4-(Phenylmethyl)-phenol & GC & Not active (TS) \\
\hline Multiple & $1806-26-4$ & 4-Octylphenol & GC & Active [28], not active (TS) \\
\hline
\end{tabular}


Table 1 (continued)

\begin{tabular}{|c|c|c|c|c|}
\hline Fraction & CAS & Name & $\mathrm{LC} / \mathrm{GC}$ & TTR-binding activity \\
\hline Multiple & $26444-49-5$ & Cresyldiphenyl phosphate & $\mathrm{GC}$ & Not active (TS) \\
\hline Multiple & $3055-96-7$ & Hexaethyleneglycol monododecyl ether & GC & Not active (TS) \\
\hline Multiple & $1338-24-5$ & Naphthenic acid & GC & Not active (TS) \\
\hline Multiple & $111-02-4$ & Squalene & GC & Not active (TS) \\
\hline Multiple & $115-96-8$ & Tris(2-chloro-ethyl) phosphate & GC & Not active (TS) \\
\hline Multiple & $78-42-2$ & Tris(2-ethylhexyl) phosphate & GC & Not active (TS) \\
\hline \multicolumn{5}{|c|}{ Additional tested compounds (not tentatively identified in the sediment sample) } \\
\hline & $80-05-7$ & Bisphenol A & & Not active (TS and [5]) \\
\hline & $13171-00-1$ & Celestolide & & Not active (TS) \\
\hline & $15323-35-0$ & Phantolide & & Not active (TS) \\
\hline & $83-66-9$ & Musk ambrette & & Not active (TS) \\
\hline & $81-14-1$ & Musk ketone & & Not active (TS) \\
\hline & $101-20-2$ & Triclocarban & & Not active (TS) \\
\hline & $15307-86-5$ & Diclofenac & & Active (TS) \\
\hline
\end{tabular}

No info no available literature on TTR-binding activity and not tested in this study, TS this study

Out of the tentatively identified compounds in the sediment sample, only two could be confirmed as TTR binders, i.e., triclosan and the technical mixture of nonylphenol. Triclosan is an antibacterial agent, which has shown to cause a dosedependent decrease in total $\mathrm{T}_{4}$ in a 4-day oral exposure study with rats [31]. TTR binding could be an explanation for this observed decrease of free circulating $\mathrm{T}_{4}$. Nonylphenol is a detergent, which is used in a technical mixture and also a degradation product of nonylphenol polyethoxylate surfactants. Eight different isomers were distinguished in the GC chromatogram, consisting of linear and differently branched isoforms typically present in a technical mixture. An earlier study confirmed that branched nonylphenol can bind to human TTR, whereas no binding was observed for linear nonylphenol [25]. Nonylphenol has been reported before as a binder to TTR from several species (Table S1), but these studies did not report which form of nonylphenol was tested. Earlier analysis of exactly the same sample reported a triclosan concentration of $26 \mathrm{ng} / \mathrm{g}$ d.w. sediment and a nonylphenol concentration of $210 \mathrm{ng} / \mathrm{g}$ d.w. [32]. Based on their REP values and concentrations, these two compounds could explain less than $1 \%$ of the observed TTR-binding potency in the unfractionated extract. Only four of the compounds in Table 1 have been studied earlier for their TTR-binding potency, i.e., the nonylphenol, two phthalates (butylbenzyl phthalate and dibutyl phthalate), and octylphenol. The phthalates and octylphenol were reported to exhibit weak TTR binding in RLBA with salmon and frog TTR [28] (Table S1). In the present study, however, these compounds showed no binding to human TTR. The four compounds present in fraction RP4NP6 are a group of natural compounds including plant steroids (Table 1). Although the $\log K_{\text {ow }}$ values are slightly too high $(\sim 8.8)$ for the corresponding fraction ( $\left.\log K_{\mathrm{ow}} 6-8\right)$, it is likely that these compounds are present in the sediment extract. The compounds are more likely to have an androgen- or estrogen-disrupting potential than TTR-binding activity, considering the structural similarities with steroid hormones.

In addition, the last seven compounds included in Table 1 were tested for their TTR-binding potency, due to their similar properties (aromatic, halogenated, and hydroxylated) as already tested compounds. Bisphenol A is a high-productionvolume chemical (HPVC) used in plastics and epoxy resins, with high concern for its endocrine-disrupting properties, though no TTR-binding activity was observed. The musk compounds, celestolide, phantolide, ambrette, and ketone, did not bind to TTR. The antiseptic agent triclocarban is a common additive in soaps and other personal care products, and also for triclocarban, no TTR-binding activity was found.

Diclofenac is a non-steroidal anti-inflammatory drug (NSAID) used at high volume and is known to pass through the wastewater treatment plants and enter the water environment [33]. Diclofenac bound to TTR with a $T_{4}$ REP value of 0.032 (Table S1). Diclofenac is also known for its binding potency to TTR from earlier studies on NSAIDs as antiamyloid compounds [34]. Anti-amyloid genesis drugs are small molecules that stabilize TTR by binding to its TH binding sites (based on an in vitro fibril formation assay). Diclofenac is aromatic and chlorinated in ortho-positions and has a carboxylic acid moiety on the second aromatic ring. The binding pocket of TTR is well defined, and the size and shape of the ligand are important for the affinity [35]. It has been shown that biphenyls, dibenzofurans, diphenylethers, stilbene, and flavone are basic structures with optimal size that can be functionalized with substituents to interact in the pocket [36]. The compounds listed in Table 1 
fit well into that description. Of the 58 compounds in Table 1 , 10 compounds are halogenated (17\%), 45 have aromatic ring systems (76\%), and 15 are hydroxylated (26\%).

\section{EDA strategy}

The identification strategy presented here is limited by several set criteria (e.g., the intensity ratio active/non-active control fraction $>100$, match factor $>70 \%$, standard availability, etc.). Another approach that currently receives significant attention is "suspect screening" using databases such as the one presented here. This strategy screens all masses but only the hits in the database are being further evaluated, which is an essential difference in comparison with the non-target screening strategy presented here. In addition, the prerequisite for the compound to be present in the NIST library limits the possibilities to identify active compounds which are metabolites and at low concentrations. Hence, future research is needed to aid the identification of the key toxicants present in the environment by developing more sensitive strategies for the chemical identification in the framework of EDA. Most crucial is the capability to exclude the presence of background compounds without losing the compounds of interest. One promising approach is the application of high-resolution fractionation techniques [37] or the application of the two-dimensional separation with a LC $\times$ LC system [38]. This together can improve the EDA efficiency regarding throughput and success rate. Still, it is important to get a better understanding and overview of chemical structures and effect-based correlations to carry out EDA in the search for key toxicants in our environment. Hopefully, the database presented here is one step in the direction for improved THDC identification.

\section{Conclusion}

Table S1 in the Electronic Supplementary Material summarizes the state of knowledge regarding the TTR-binding capacity of a wide range of chemical compounds, e.g., environmental contaminants, pesticides, pharmaceuticals, metabolites, etc. The table is presented to facilitate future EDA studies to identify TTR binders and non-binders. Here, we have demonstrated an identification strategy in identifying THDCs in a sediment extract with TTR-binding potency. Two compounds, triclosan and nonylphenol technical mixture, could be confirmed to have contributed to the observed activity in the TTR-binding assay; however, only $1 \%$ of the activity could be explained by the presence of these two compounds. The chemical properties in Table S1 are biased towards aromatic, halogenated, and hydroxylated compounds, which are structural features of the natural hormone thyroxine. The PCA illustrated this in the first PCs which displayed a separation of aromatic and halogenated compounds (active and non-active) from fatty acids (non-active) and perfluorinated compounds (active). In addition, the database presented here was used to further evaluate the structure relationship with the TTR-binding activity and a model was developed to predict the potential of contaminants to bind to TTR [39]. Here, it is demonstrated that more data on TTRbinding compounds is needed to cover the potential binding potency of compounds without these typical characteristics. Also, more sensitive identification strategies combining, e.g., novel fractionation techniques and accurate chemical analysis instruments are needed, without increasing the already substantial workload on the identification step.

Acknowledgments This work was performed within the framework of the EU-supported program MODELKEY (Contract No. 511237 (GOCE)), the Marie Curie Research Training Network KEYBIOEFFECTS (MRTNCT-2006-035695), and the Swedish Research Council (FORMAS)supported project MiSSE (210-2012-131).

Open Access This article is distributed under the terms of the Creative Commons Attribution 4.0 International License (http:// creativecommons.org/licenses/by/4.0/), which permits unrestricted use, distribution, and reproduction in any medium, provided you give appropriate credit to the original author(s) and the source, provide a link to the Creative Commons license, and indicate if changes were made.

\section{References}

1. WHO/UNEP (2013) State of the science of endocrine disrupting chemicals-2012. Geneva, Switzerland

2. OECD (2014) New scoping document on in vitro and ex vivo assays for the identification of modulators of thyroid hormone signalling. Series on Testing and Assessment, vol ENV/JM/MONO(2014)23

3. OECD (2006) Detailed review paper on thyroid hormone disruption assays. Series on testing and assessment, vol ENV/JM/ MONO(2006)24

4. Lans MC, Klasson-Wheler E, Willemsen M, Meussen E, Safe S, Brouwer A (1993) Structure-dependent, competitive interaction of hydroxy-polychlorobiphenyls, -dibenzo- $p$-dioxins and dibenzofurans with human transthyretin. Chem-Biol Interaction 88:7-21

5. Meerts IATM, van Zanden JJ, Luijks EAC, van Leeuwen-Bol I, Marsh G, Jakobsson E, Bergman Å, Brouwer A (2000) Potent competitive interactions of some brominated flame retardants and related compounds with human transthyretin in vitro. Toxicol Sci 56:95-104

6. Murk AJ, Rijntjes E, Blaauboer BJ, Clewell R, Crofton KM, Dingemans MML, Furlow JD, Kavlock R, Koehrle J, Opitz R, Traas T, Visser TJ, Xia M, Gutleb A (2013) Mechanism-based testing strategy using in vitro approaches for identification of thyroid hormone disrupting chemicals. Toxicol in Vitro 27(4): 1320-1346

7. Ucan-Marin F, Arukwe A, Mortensen A, Gabrielsen GW, Fox GA, Letcher RJ (2009) Recombinant transthyretin purification and competitive binding with organohalogen compounds in two gull species 
(Larus argentatus and Larus hyperboreus). Toxicol Sci 107(2): 440-450

8. Power DM, Elias NP, Richardson SJ, Mendes J, Soares CM, Santos CRA (2000) Evolution of the thyroid hormone-binding protein, transthyretin. Gen Comp Endocrin 119:241-255

9. Meerts IATM, Assink Y, Cenijn PH, Van den Berg JHJ, Weijers BM, Bergman Å, Koeman JH, Brouwer A (2002) Placental transfer of a hydroxylated polychlorinated biphenyl and effects on fetal and maternal thyroid hormone homeostasis in the rat. Toxicol Sci 68: 361-371

10. Hamers T, Kamstra JH, Sonneveld E, Murk AJ, Visser TJ, van Velzen MJM, Brouwer A, Bergman $\AA$ (2008) Biotransformation of brominated flame retardants into potentially endocrine-disrupting metabolites, with special attention to 2,2',4,4'-tetrabromodiphenyl ether (BDE-47). Mol Nutr Food Res 52(2):284-298

11. Purkey H, Palaninathan SK, Kent KC, Smith C, Safe SH, Sacchettini JC, Kelly JW (2004) Hydroxylated polychlorinated biphenyls selectively bind transthyretin in blood and inhibit amyloidogenesis: rationalizing rodent PCB toxicity. Chem Biol 11(12):1719-1728

12. Weiss JM, Andersson PL, Lamoree MH, Leonards PEG, van Leeuwen SPJ, Hamers T (2009) Competitive binding of perfluorinated compounds to the thyroxine transport protein transthyretin. Toxicol Sci 109(2):206-216

13. Grung M, Lichtenthaler R, Ahel M, Tollefsen KE, Langford K, Thomas KV (2007) Effects-directed analysis of organic toxicants in wastewater effluent from Zagreb, Croatia. Chemosphere 67: $108-120$

14. Thomas KV, Hurst MR, Matthiessen P, Waldock MJ (2001) Characterization of estrogenic compounds in water samples collected from United Kingdom estuaries. Environ Toxicol Chem 20(10): $2165-2170$

15. Thomas KV, Hurst MR, Matthiessen P, McHugh M, Smith A, Waldock MJ (2002) An assessment of in vitro androgenic activity and the identification of environmental androgens in United Kingdom estuaries. Environ Toxicol Chem 21(7):1456-1461

16. Weiss JM, Simon E, Stroomberg G, De Boer R, De Boer J, Van der Linden S, Leonards PEG, Lamoree MH (2011) Identification strategy for unknown pollutants using high resolution mass spectrometry: androgen disrupting compounds identified through effect-directed analysis. Anal Bioanal Chem 400:3141-3149

17. Thomas KV, Thain JE, Waldock MJ (1999) Identification of toxic substances in United Kingdom estuaries. Environ Sci Technol 18(3):401-411

18. Houtman CJ, Cenijn PH, Hamers T, Lamoree MH, Legler J, Murk AJ, Brouwer A (2004) Toxicological profiling of sediment using in vitro bioassays, with emphasis on endocrine disruption. Environ Toxicol Chem 23(1):32-40

19. Houtman CJ, Booij P, Jover E, Pascual del Rio D, Swart K, van Velzen M, Vreuls R, Legler J, Brouwer A, Lamoree MH (2006) Estrogenic and dioxin-like compounds in sediment from Zierikzee harbour identified with CALUX assay-directed fractionation combined with one and two dimensional gas chromatography analyses. Chemosphere 65:2244-2252

20. Grote M, Brack W, Altenburger R (2005) Identification of toxicants from marine sediment using effect-directed analysis. Environ Toxicol 20(5):475-486

21. Houtman CJ, Van Oostveen AM, Brouwer A, Lamoree MH, Legler J (2004) Identification of estrogenic compounds in fish bile using bioassay-directed fractionation. Environ Sci Technol 38:6415-6423

22. Brack W (2003) Effect-directed analysis: a promising tool for the identification of organic toxicants in complex mixtures. Anal Bioanal Chem 377:397-407
23. Lübke-von Varel U, Machala M, Ciganek M, Neca J, Pencikova K, Palkova L, Vondracek J, Löffler I, Streck G, Reifferscheid G, Flückiger-Isler S, Weiss JM, Lamoree MH, Brack W (2011) Polar compounds dominate in vitro effects of sediment extracts. Environ Sci Technol 45:2384-2390

24. Suzuki G, Takigami H, Nose K, Takahashi S, Asari M, Sasaki S (2007) Dioxin-like and transthyretin-binding compounds in indoor dusts collected from Japan: average daily dose and possible implications for children. Environ Sci Technol 41:1487-1493

25. Simon E, van Velzen M, Brandsma SH, Lie E, Løken K, de Boer J, Bytingsvik J, Jenssen BM, Aars J, Hamers T, Lamoree MH (2013) Effect-directed analysis to explore the polar bear exposome: identification of thyroid hormone disrupting compounds in plasma. Environ Sci Technol 47(15):8902-8912

26. Weiss JM, Hamers T, Thomas KV, Van der Linden S, Leonards PEG, Lamoree MH (2009) Masking effect of anti-androgens on androgenic activity in European river sediment unveiled by effectdirected analysis. Anal Bioanal Chem 394:1385-1397

27. Ucan-Marin F, Arukwe A, Mortensen A, Gabrielsen GW, Letcher RJ (2010) Recombinant albumin transport proteins from two gull species and human: chlorinated and brominated contaminant binding and thyroid hormones. Environ Sci Technol 44(1):497-504

28. Ishihara A, Sawatsubashi S, Yamauchi K (2003) Endocrine disrupting chemicals: interference of thyroid hormone binding to transthyretins and to thyroid hormone receptors. Mol Nutr Food Res 199:105-117

29. Prapunpoj P (2009) Evolutionary changes to transthyretin. FEBS 276(19):5329-5718

30. Hamers T, Kamstra JH, Sonneveld E, Murk AJ, Kester MHA, Andersson PL, Legler J, Brouwer A (2006) In vitro profiling of the endocrine-disrupting potency of brominated flame retardants. Toxicol Sci 92(1):157-173

31. Crofton KM, Paul KB, DeVito MJ, Hedge JH (2007) Shortterm in vivo exposure to the water contaminant triclosan: evidence for disruption of thyroxine. Environ Toxicol Pharmacol 24:194-197

32. Schmitt C, Streck G, Lamoree MH, Leonards PEG, Brack W, De Deckere E (2010) Effect directed analysis of riverine sedimentsthe usefulness of Potamopyrgus antipodarum for in vivo effect confirmation of endocrine disruption. Aquat Toxicol 101(1): 237-243

33. Singer H, Jaus S, Hanke I, Lueck A, Hollender J, Alder AC (2000) Determination of biocides and pesticides by on-line solid phase extraction coupled with mass spectrometry and their behaviour in wastewater and surface water. Environ Poll 158:3054-3064

34. Palaninathan SK, Mohamedmohaideen NN, Orlandini E, Ortore G, Nencetti S, Lapucci A, Rossello A, Freundlich JS, Sacchettini JC (2009) Novel transthyretin amyloid fibril formation inhibitors: synthesis, biological evaluation, and X-ray structural analysis. PLoS One 4(7):e6290

35. Prapunpoj P, Leelawattwattana L (2009) Evolutionary changes to transthyretin: structure-function relationships. FEBS 276:53305341

36. Baures PW, Peterson SA, Kelly JW (1998) Discovering transthyretin amyloid fibril inhibitors by limited screening. Bioorgan Med Chem 6:1389-1401

37. Booij P, Vethaak AD, Leonards P, Sjollema SB, Kool J, De Voogt P, Lamoree MH (2014) Inhibition of photosynthesis inhibitors of pelagic marine algae using 96-well plate micro-fractionation for enhanced through-put in effect-directed analysis. Environ Sci Technol 48:8003-8011

38. Ouyang X, Leonards P, Legler J, van der Oost R, de Boer J, Lamoree M (2015) Comprehensive two-dimensional liquid chromatography coupled to high resolution time of flight mass 
spectrometry for chemical characterization of sewage treatment plant effluents. J Chromatogr A 1380:139-145. doi:10.1016/j. chroma.2014.12.075

39. Zhang J, Kamstra JH, Ghorbbanzadeh M, Weiss JM, Hamers T, Andersson PL (2015) An in silico approach to identify potential thyroid hormone disruptors among currently known dust contaminants and their metabolites. Submitted to Environmental Science \& Technology

40. Morgado I, Hamers T, van der Veen L, Power DM (2007) Disruption of thyroid hormone binding to sea bream recombinant transthyretin by ioxinyl and polybrominated diphenyl ethers. Chemosphere 69:155-163 